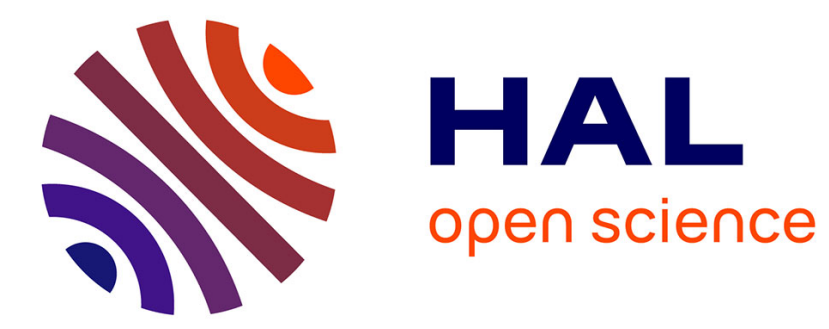

\title{
EPISTASIS, PLEIOTROPY, AND THE MUTATION LOAD IN SEXUAL AND ASEXUAL POPULATIONS
}

Denis Roze, Alexandre Blanckaert

\section{To cite this version:}

Denis Roze, Alexandre Blanckaert. EPISTASIS, PLEIOTROPY, AND THE MUTATION LOAD IN SEXUAL AND ASEXUAL POPULATIONS. Evolution - International Journal of Organic Evolution, 2014, 68, pp.137-149. 10.1111/evo.12232 . hal-01137848

\section{HAL Id: hal-01137848 \\ https://hal.science/hal-01137848}

Submitted on 31 Mar 2015

HAL is a multi-disciplinary open access archive for the deposit and dissemination of scientific research documents, whether they are published or not. The documents may come from teaching and research institutions in France or abroad, or from public or private research centers.
L'archive ouverte pluridisciplinaire HAL, est destinée au dépôt et à la diffusion de documents scientifiques de niveau recherche, publiés ou non, émanant des établissements d'enseignement et de recherche français ou étrangers, des laboratoires publics ou privés. 
Epistasis, pleiotropy and the mutation load in sexual and asexual populations

Denis Roze ${ }^{1,2}$ and Alexandre Blanckaert ${ }^{3}$

1- CNRS, UMR 7144, Adaptation et Diversité en Milieu Marin, 29682 Roscoff, France 2- UPMC Université Paris VI, 29682 Roscoff, France

3- Mathematics and Biosciences Group, Department of Mathematics, University of Vienna, 1090 Vienna, Austria

Corresponding author: Denis Roze

Station Biologique de Roscoff

Place Georges Teissier, CS90074

29688 Roscoff Cedex, France

Ph: (+33) 2982923 20; fax: (+33) 298292324

roze@sb-roscoff.fr

Running head: Fitness landscapes and the mutation load 
2 Mutation may impose a substantial load on populations, which varies accord3 ing to the reproductive mode of organisms. Over the last years, different authors used 4 adaptive landscape models to predict the long term effect of mutation on mean fitness; 5 however, many of these studies assumed very weak mutation rates, so that at most 6 one mutation segregates in the population. In this paper we derive several simple 7 approximations (confirmed by simulations) for the mutation load at high mutation 8 rate $(U)$, using a general model that allows us to play with the number of selected 9 traits $(n)$, the degree of pleiotropy of mutations and the shape of the fitness function ${ }_{10}$ (which affects the average sign and magnitude of epistasis among mutations). When 11 mutations have strong fitness effects, the equilibrium fitness $\bar{W}$ of sexuals and asexu12 als is close to $e^{-U}$; under weaker mutational effects, sexuals reach a different regime ${ }_{13}$ where $\bar{W}$ is a simple function of $U$ and of a parameter describing the shape of the 14 fitness function. Contrarily to weak-mutation results showing that $\bar{W}$ is an increasing 15 function of population size and a decreasing function of $n$, these parameters may have opposite effects in sexual populations at high mutation rate.

17

18 Keywords: adaptive landscape, epistasis, evolutionary quantitative genetics, multi19 locus models, mutation load, stabilizing selection 
Although mutation represents the ultimate fuel for adaptation, it is also the

source of a fitness cost for populations due to the production of sub-optimal genotypes. This "mutation load" may in turn affect many important evolutionary processes, such as the evolution of sex and recombination (Kondrashov, 1988; Barton,

${ }_{41}$ They showed that when interactions among mutations tend to reinforce their deleteri42 ous effect (negative epistasis) the mean fitness of sexual populations increases, while it 
decreases when deleterious alleles tend to compensate each other (positive epistasis).

By contrast, the mean fitness of asexual populations is not affected by epistasis and remains approximately $e^{-U}$.

In a different class of models, mutations affect a given number of phenotypic traits which in turn influence fitness. Fisher (1930), Haldane (1932) and Wright (1935) considered different models of stabilizing selection acting on a single quantitative trait influenced by many genes. In particular, Haldane and Wright derived expressions for equilibrium allele frequencies, from which mean fitness can be deduced; further developments (to which we will return below) were done in the sixties and seventies, in particular by Kimura $(1965)$, Bulmer $(1971,1972)$ and Lande $(1976,1977)$. Interestingly, compensatory effects between mutations emerge naturally from this type of model (a mutation that displaces from the optimum can be compensated by another mutation bringing closer to the optimum), together with distributions of epistatic interactions among mutations. In his Genetical Theory of Natural Selection, Fisher also proposed a model involving multiple phenotypic traits in support of his idea that adaptation is mainly due to mutations of small effect: this geometrical model represents an $n$-dimensional phenotypic space where each dimension corresponds to the value of a quantitative trait, and where mutations correspond to random vectors displacing individuals in phenotypic space. Fitness is assumed to decrease monotonously as the Euclidean distance from a point corresponding to the optimal phenotype increases. Although the initial goal of Fisher's geometrical model was to describe the dynamics ${ }^{4}$ of adaptation (Fisher, 1930; Orr, 1998, 2000; Welch and Waxman, 2003), it has also ${ }_{5}$ been used to explore the effects of various parameters (population size, number of 6 phenotypic traits under selection, shape of the fitness peak...) on the mean fitness 
of populations in equilibrium situations. Several authors considered a weak-mutation limit $(N U \ll 1$, where $N$ is population size and $U$ the total rate of mutations affecting the traits under selection) so that at most one mutation segregates in the population at a given time (Hartl and Taubes, 1998; Poon and Otto, 2000; Sella and Hirsh, 2005; Tenaillon et al., 2007; Sella, 2009): the population evolves away from the optimum by fixing weakly deleterious alleles by random drift, and occasionally returns near the optimum by fixing a compensatory mutation. For example, using the fitness function $W=\exp \left(-d^{Q}\right)$, where $d$ is the Euclidean distance from the optimum in phenotypic space and $Q$ a parameter affecting the shape of the fitness peak, Tenaillon et al. (2007) showed that the equilibrium mean fitness of a haploid population is approximately $(1-1 /(2 N))^{\frac{n}{Q}}$, where $n$ is the number of traits affecting fitness (often called "complexity"). Note that this result does not depend on the reproductive mode of organisms (sexual or asexual) since at most one locus is polymorphic in this low mutation limit.

It seems likely that in many organisms, however, $N U \gg 1$, in which case many of the loci affecting the traits under selection may be polymorphic at the same time. In this regime, and assuming that the position of the optimum remains constant over time, the population can be represented by a collection of points centered around the optimum, while mean fitness can usually be expressed in terms of the variance of the different trait values in the population. Since the original works of Haldane (1932) and Wright (1935), numerous models have been used to calculate the genetic variance of a quantitative trait (or a set of traits) maintained at mutation-selection balance, and different regimes have been described (e.g., Bulmer, 1989; Bürger, 2000; Johnson and Barton, 2005; Zhang and Hill, 2005). When mutations tend to have strong fitness 
effects, the distribution of phenotypic values is often highly leptokurtic (with a sharp peak at the optimum) and may present a singularity (Waxman and Peck, 1998). In the limit of very strong selection, the expected contribution to future generations of individuals located away from the optimum becomes negligible; in this case, one expects that mean fitness, relative to the fitness of individuals at the optimum $\left(\bar{W} / W_{0}\right)$ should be approximately $e^{-U}$ when the number of new mutations per individual is Poisson-distributed, independently of the exact shape of the fitness peak. Indeed, a recursion for the frequency of individuals at the optimum $\left(x_{0}\right)$ is given by $x_{0}{ }^{\prime}=$ $e^{-U} x_{0} W_{0} / \bar{W}$ ( $e^{-U}$ being the probability that no mutation occurs), which yields the desired result when $x_{0}^{\prime}=x_{0}$ (e.g., Kimura and Maruyama, 1966).

When mutations tend to have weak fitness effects, different results can be obtained by assuming that equilibrium distributions of phenotypic values are Gaussian. Most models exploring this regime considered a single trait under selection and a Gaussian (or quadratic) fitness function, although multivariate models have also been proposed (Lande, 1980; Turelli, 1985). Different assumptions regarding the genetic architecture of traits (in particular the number of alleles per locus) have been explored. Continuum-of-alleles models assume that an infinite number of alleles (having different phenotypic effects) segregate at each locus; expressions for the genetic variance and mean fitness under this scenario have been obtained by assuming that the equilibrium distribution of allelic effects in the population is Gaussian at each locus (e.g., Kimura, 1965; Lande, 1976). Lande (1980) generalized these models to the case where selection acts on multiple traits, assuming a multivariate Gaussian fitness function. Although the general expression for equilibrium genetic variances (and mean fitness) is cumbersome, it takes a simpler form when selection acts independently on each trait, and in 
the absence of correlation of mutational effects across traits (p.294 in Bürger, 2000).

Neglecting linkage disequilibria among loci, mean fitness can then be written as a simple function that depends on the number of selected traits, number of loci, strength of selection and mutational variance (see Results section below). Other models considered stabilizing selection (Gaussian fitness function) acting on a single trait coded by multiple biallelic loci (e.g., Bulmer, 1972, 1985; Barton, 1986, 1989). As shown by Barton (1986), multiple possible equilibria exist for allele frequencies at the different loci. Although the population mean phenotype may deviate from the optimum at some of these equilibria, Barton (1989) argues that perturbations generated for example by random drift should keep the population mean near the optimum. Neglecting linkage disequilibria, and as long as the effects of mutation and drift at each locus are weaker than selection, one obtains from the expression of the equilibrium genetic variance that mean fitness should be close to $e^{-U}$. More recently, Zhang and Hill (2003) investigated the maintenance of genetic variation in multiple-trait models (including correlations among traits), assuming that the fitness function is multivariate Gaussian, and that selection at each locus is sufficiently strong so that mutations remain rare in the population (only one mutant allele segregates at each locus). Results for the case of a changing optimum have been derived by Zhang (2012). Finally, effects of linkage disequilibria have been explored in both continuum-of-alleles and biallelic models, for the case of a single trait under Gaussian stabilizing selection (Bulmer, 1974; Lande, 1976; Turelli and Barton, 1990), and were shown to be minor as long as the total rate of mutation on loci affecting the trait remains small, and linkage is not too tight.

In this article, we derive several simple approximations for the mutation load in both sexual and asexual populations at high mutation rate, using a general model that 
allows us to play with the shape of the fitness peak (which in turn affects the average sign and magnitude of epistasis among mutations), the number of traits under selection and the degree of pleiotropy of mutations. These results are tested by individualbased simulations representing stabilizing selection on traits coded by large numbers of loci. As we will see, two different regimes are observed in asexual populations: when mutations tend to have large fitness effects, mean fitness only depends on the mutation rate and is close to $e^{-U}$, while under weaker mutational effects mean fitness increases as mutational effects decreases, and can be expressed in terms of the mutation rate, the dimensionality of the landscape, the shape of the fitness peak and the average deleterious effect of mutations. By contrast, three regimes are observed in the case of sexual populations: under strong mutational effects, mean fitness is again close to $e^{-U}$, while it switches to a different value that only depends on the mutation rate and the shape of the fitness peak as mutational effects decrease. Interestingly, mean fitness is the same under these two regimes when the fitness function is Gaussian $\left(\bar{W} \approx e^{-U}\right)$, while $\bar{W}>e^{-U}$ in the second regime when the fitness peak is flatter than a Gaussian around the optimum, and $\bar{W}<e^{-U}$ when the fitness peak is sharper. Finally, as mutational effects continue decreasing a third regime is entered, where allele frequency dynamics are dominated by mutation and drift. We also obtain expressions for linkage disequilibria and find that they can have a substantial effect on fitness, in particular when the mutation rate is high and the number of traits under selection is low. 
Multivariate stabilizing selection. Parameters and variables are summarized in

Table 1. The model represents an infinite haploid population with discrete generations, under stabilizing selection on $n$ phenotypic traits; for each individual, trait values are given by the vector $\mathbf{z}=\left(z_{0}, z_{1}, \ldots, z_{n}\right)$. Following others (Wilke and Adami, 2001; Tenaillon et al., 2007; Gros et al., 2009), we assume for simplicity that the fitness function is spherically symmetric, and is given by:

$$
W=\exp \left[-\frac{d^{Q}}{2 V_{\mathrm{S}}}\right]
$$

where $d=\sqrt{\sum_{i=1}^{n} z_{i}^{2}}$ is the Euclidean distance in phenotypic space from the optimal phenotype (located at $z_{i}=0$ for each trait $i$ ), $V_{\mathrm{S}}$ measures the strength of selection, while the parameter $Q$ determines the shape of the fitness peak: $Q=2$ corresponds to a (multivariate) Gaussian fitness function, while $Q<2$ (resp. $Q>2$ ) leads to a sharper (resp. flatter) fitness peak. Traits are coded by $L$ biallelic loci, each locus affecting a subset $m \leq n$ of phenotypic traits (sampled randomly and independently for each locus among the set of $n$ traits). As in Lourenço et al. (2011), the parameter $m$ thus measures the number of traits affected by a single mutation ("mutation pleiotropy"), while $n$ is the total number of traits under selection ("complexity"): $m=n$ corresponds to maximal pleiotropy, where each mutation affects all selected traits (as in Fisher's model). The genomic mutation rate is denoted $U$, so that each locus mutates at rate $u=U / L$. For simplicity, we assume additive effects of the different loci on phenotypic traits, and no environmental variance. Denoting 0 and 1 the two alleles at each locus, and $X_{j}$ an indicator variable that equals 0 if an individual carries allele 0 at locus $j$, 
and 1 otherwise, the value of trait $i$ in a given individual is given by:

$$
z_{i}=\sum_{j=1}^{L} r_{i j} X_{j}
$$

where $r_{i j}$ measures the effect of allele 1 at locus $j$ on trait $i$ : an individual carrying alleles 0 at all loci is thus at the phenotypic optimum. For each locus $j$, the effect of allele 1 on each of the $m$ traits affected by the locus is sampled in a Gaussian distribution with mean 0 and variance $a^{2}$ (therefore, $r_{i j}$ can be positive or negative). Finally, we denote $\bar{s}$ the mean deleterious effect of mutations (measured on log-fitness) in a population at the optimum, given by Gros et al. (2009):

$$
\bar{s}=\frac{\left(2 a^{2}\right)^{\frac{Q}{2}}}{2 V_{\mathrm{S}}} \frac{\Gamma\left(\frac{m+Q}{2}\right)}{\Gamma\left(\frac{m}{2}\right)}
$$

(where $\Gamma$ is Euler's gamma function), which simplifies to $\bar{s}=\frac{m}{2}\left(a^{2} / V_{\mathrm{S}}\right)$ in the case of a Gaussian fitness function $(Q=2$, in agreement with Martin and Lenormand, 2006). As shown by Gros et al. (2009), the average epistasis among pairs of mutations (at the optimum, and measured on log-fitness) is given by $\bar{e}=\left(2-2^{Q / 2}\right) \bar{s}$, which equals zero when $Q=2$ (see also ref. Martin et al., 2007) but it positive for $Q<2$, and negative for $Q>2$.

Finally, we can note that the strength of selection $V_{\mathrm{S}}$ (equation 1) can be considered as a scaling parameter: using the scaled variables $z_{i, \mathrm{~s}}=z_{i} /\left(2 V_{\mathrm{S}}\right)^{\frac{1}{Q}}$ to measure phenotypic traits, fitness in terms of $z_{i, \mathrm{~s}}$ variables becomes independent of $V_{\mathrm{S}}$, while the variance of the distribution of mutational effects on each $z_{i, \mathrm{~s}}$ equals $a_{\mathrm{s}}^{2}=a^{2} /\left(2 V_{\mathrm{S}}\right)^{\frac{2}{Q}}$ : therefore, $a^{2}$ and $V_{\mathrm{S}}$ affect the results only through the compound parameter $a_{\mathrm{s}}^{2}$. In the simulations (described next), we used $V_{\mathrm{S}}=1 / 2$ so that $a_{\mathrm{s}}^{2}=a^{2}$. As shown by equation 3, large (resp. small) values of $a_{\mathrm{s}}^{2}$ imply that mutations have strong (resp. weak) fitness effects. 
Simulations. Analytical predictions are tested using individual-based simulations.

Our program (written in $\mathrm{C}++$, and available upon request) represents a haploid population of $N$ individuals with discrete generations. The genome of each individual is represented by a sequence of $L$ bits ( 0 or 1 ) corresponding to the different loci. Phenotypic effects of mutation at each locus are sampled at the start of the simulation: the $m$ traits affected by a given locus are sampled randomly (and independently for each locus) among the $n$ selected traits, and the effect of mutation on each of these $m$ traits is sampled from a Gaussian distribution with mean 0 and variance $a^{2}$. At the start of a generation, phenotypic values are computed for each individual; from this, one obtains the fitness of the individual based on equation 1. For each individual of the next generation, two parents are sampled (the probability that an individual is sampled being proportional to its fitness); selfing is allowed if the same individual is sampled twice. To generate a recombinant chromosome, the number of cross-overs is sampled from a Poisson distribution with parameter $R$ (genome map length), and the position of each cross-over along the chromosome is sampled from a uniform distribution ( $R=0$ corresponds to the case of an asexual population). Finally, the number of new mutations occurring in each genome is sampled from a Poisson distribution with parameter $U$, and the positions of mutant loci are sampled randomly; alleles are switched at mutant loci, from 0 to 1 or from 1 to 0 (mutation and back mutation thus occur at the same rate). In order to gain execution speed, the program is parallelized (using the MPI library) to run on several processors, each processor dealing with a given segment of the genome, for all individuals; execution speed can be considerably increased when the number of processors is sufficiently large so that the probability that an event (mutation or cross-over) occurs in a given segment per generation is 
low (in which case most genome segments stay unchanged from one generation to the next).

At the start of the simulation each genome contains only "0" alleles, which corresponds to the fitness optimum. Simulations run for 50000 generations, equilibrium being reached during the first 30000 generations for most parameter values tested. Every 1000 generations, the program records the mean fitness of the population and the first 6 moments of the distribution of each phenotypic trait in the population. The results shown in the different figures correspond to averages over the last 20000 generations. Error bars were calculated using Hastings' (1970) batching method, dividing these 20000 generations into 4 batches of 5000 generations and calculating the standard error over these 4 batches; however, error bars were generally small relative to the size of symbols used in the figures and are thus not shown. All our simulation results (with the Mathematica commands used to generate the figures) can be found in the Supplementary Material.

\section{RESULTS}

Asexual population. Figure 1 shows the equilibrium mean fitness of an asexual population as a function of the variance of mutational effects (on scaled traits) $a_{\mathrm{s}}^{2}$ (left) or of the mean deleterious effect of mutations $\bar{s}$ (right), and for different values of the shape parameter $Q$. As $a_{\mathrm{s}}^{2}$ becomes large, mean fitness converges to $e^{-U}$ (which is close to 0.6 for $U=0.5$, dashed line). For smaller values of the scaled mutational variance, however, the population reaches a different regime where $\bar{W}>e^{-U}$. In this case, an approximation for mean fitness can be obtained by assuming that distributions 
of phenotypic traits $z_{i}$ are Gaussian at equilibrium. As explained in Supplementary

File S1, Euclidean distances from the optimum $(d)$ then follow a $\chi$-distribution, which yields:

$$
\overline{\ln W}=-\frac{\left(2 V_{\mathrm{G}}\right)^{\frac{Q}{2}}}{2 V_{\mathrm{S}}} \frac{\Gamma\left(\frac{n+Q}{2}\right)}{\Gamma\left(\frac{n}{2}\right)}
$$

where $V_{\mathrm{G}}$ is the genetic variance at equilibrium (the variance of $z_{i}$, which by symmetry should be the same for all traits $i$ ); for $Q=2$ (Gaussian fitness function), this simplifies to $\overline{\ln W}=-(n / 2)\left(V_{\mathrm{G}} / V_{\mathrm{S}}\right)$. An expression for $V_{\mathrm{G}}$ at equilibrium is derived in Supplementary File S1. Assuming that $\overline{\ln W} \approx \ln \bar{W}$, one obtains that when $m=n$ (that is, when each mutation affects all phenotypic traits):

$$
\bar{W} \approx \exp \left[-\bar{s}\left(\frac{n U}{\bar{s} Q}\right)^{\frac{Q}{2+Q}}\right]
$$

When $Q=2$, equation 5 simplifies to $\exp \left[-\sqrt{\frac{n}{2} U \bar{s}}\right]$, in agreement with the result obtained from Lande's analysis (1980) when selection acts independently on the different traits, and in the absence of mutational covariances among traits (that is, when the variance-covariance matrices representing the effects of selection and mutation on the traits are diagonal). Indeed, in an asexual population the whole genome can be considered as a single locus with many alleles (provided that the number of loci is sufficiently large), in which case Lande's analysis of the continuum-of-alleles model yields $V_{\mathrm{G}}=\sqrt{U a^{2} V_{\mathrm{S}}}$ when mutation and selection covariance matrices are diagonal (the equilibrium genetic variance for each trait is not affected by the other traits under selection, see also p. 294 in ref. Bürger, 2000). When $m<n$, equation 5 still holds when the fitness function is Gaussian $(Q=2)$, while $\bar{W}$ is given by a slightly more complicated expression when $Q \neq 2$ (Supplementary File S1). However, this expression only depends weakly on $m$ (for fixed $\bar{s}$ ) and remains close to equation 5 in most 
cases (unless $Q$ is high and $m$ is small). As shown by Figure 1, equation 5 provides accurate predictions for $\bar{W}$ as long as $a_{\mathrm{s}}^{2}$ is not too high. Supplementary Figure S1 shows the genetic variance $V_{\mathrm{G}}$ at equilibrium, for the same parameter values as in Figure 1.

Sexual population. Figure 2 shows the equilibrium mean fitness of a sexual population as a function of $a_{\mathrm{s}}^{2}$ and $\bar{s}$. When mutations have strong effects on fitness, one obtains again that $\bar{W} \approx e^{-U}$ (right-most points on Figure 2$)$. As $a_{\mathrm{s}}^{2}$ decreases, a second regime is entered where $\bar{W}$ reaches a new value which is independent of $a_{\mathrm{s}}^{2}$, but depends on the shape parameter $Q$ (roughly for $-4<\log _{10}\left(a_{\mathrm{s}}^{2}\right)<-2$ ). Finally, as $a_{\mathrm{s}}^{2}$ continues decreasing a third regime is entered where $\bar{W}$ increases up to $\bar{W} \approx 1$ as $a_{\mathrm{s}}^{2}$ decreases. In Supplementary File S2, we show that approximations for mean fitness under these last two regimes ( $a_{\mathrm{s}}^{2}$ small) can be obtained by assuming that distributions of phenotypic values in the population are Gaussian. As in previous models (e.g., Bulmer, 1972; Barton, 1986) one obtains that the equilibrium frequency of allele 1 at locus $j\left(p_{j}\right)$ is either (i) at $p_{j}=1 / 2$ (when selection at locus $j$ is weak relative to mutation) or (ii) at one of the two symmetric equilibria where $p_{j}\left(1-p_{j}\right)=X<1 / 4$ (where $X$ is a function of the model's parameters), the locus being closer to fixation (for allele 0 or 1) as selection increases. Assuming that all loci are at equilibrium (ii) and neglecting linkage disequilibria among loci, one obtains the following approximation for mean fitness:

$$
\bar{W} \approx \exp \left[-\frac{2 U}{Q}\right]
$$

290 Figure 2 shows that this expression does indeed provide a correct prediction of $\bar{W}$ for intermediate values of $a_{\mathrm{s}}^{2}$ (dashed lines). A better approximation can be obtained by 
taking into account effects of the linkage disequilibria, which is done in Supplementary File S2 using the methods developed by Turelli and Barton (1990). As described before (Bulmer, 1971, 1974; Lande, 1976; Turelli and Barton, 1990), stabilizing selection tends to generate negative covariances among loci, which affect $\bar{W}$ through two effects: a reduction of the genetic variance $V_{\mathrm{G}}$ which directly increases $\bar{W}$, and an effect on equilibrium allele frequencies and thus on the genic variance $V_{\mathrm{g}}$ (see Appendix A) which has the opposite effect on $\bar{W}$. As long as linkage disequilibria remain small the second effect predominates, and one obtains the following approximation for $\bar{W}$ (assuming that the number of loci $L$ is large):

$$
\bar{W} \approx \exp \left[-\frac{2 U}{Q}\left(1+\frac{2 U}{n}\left(\frac{1}{r_{\mathrm{H}}}-1\right)\right)\right]
$$

where $r_{\mathrm{H}}$ is the harmonic mean recombination rate between all pairs of loci (derived in Appendix B under our simulated genetic architecture). Figure 2 shows that equation 7 does indeed provide a slightly better approximation than equation 6 for intermediate values of $a_{\mathrm{s}}^{2}$ (horizontal solid lines). Interestingly, Supplementary Figure S1 shows that $Q$ has only a weak effect on the equilibrium genetic variance $V_{\mathrm{G}}$ in this intermediate regime, which remains close to the value obtained for $Q=2$, neglecting linkage disequilibria: $V_{\mathrm{G}}=2 U V_{\mathrm{S}} / n$ (which is also obtained from house-of-cards models assuming a Gaussian fitness function, e.g. Turelli, 1985; Bürger, 2000). Finally, when $a_{\mathrm{s}}^{2}$ is very small (so that mutations have very weak fitness effects) one predicts that most loci should be at the equilibrium where $p_{j}=1 / 2$, in which case $\bar{W} \approx \exp \left[-\bar{s}(L / 4)^{\frac{Q}{2}}\right]$ where $L$ is the total number of loci. However, in this regime we expect that the relative effect of genetic drift on allele frequency dynamics could be important. In Supplementary File S4, we show that when selection at each locus becomes weaker 
than mutation and drift, an approximation for mean fitness is given by:

$$
\bar{W} \approx \exp \left[-\bar{s}\left(\frac{N U}{1+4 N u}\right)^{\frac{Q}{2}}\right]
$$

where $N$ is population size. Figure 2 shows that equation 8 provides accurate predictions when $a_{\mathrm{s}}^{2}$ is very small (left part of the figures). Furthermore, combining equations 7 and 8 (solid curves) gives good predictions for all values of $a_{\mathrm{s}}^{2}$ as long as they remain small (roughly, $<10^{-2}$ for the parameter values of Figure 2) so that the Gaussian approximation holds. For higher values of $a_{\mathrm{s}}^{2}$, simulations indicate that distributions of phenotypic values depart from Gaussian distributions: in particular, the fourth and sixth cumulants $K_{4}$ and $K_{6}$ are positive and increase as $a^{2}$ increases (while the third and fifth cumulants stay close to zero), for all values of $Q$ (results not shown). In Supplementary File S3, we show that using a Gram-Charlier expansion to approximate the distribution of phenotypic effects yields an expression for $\bar{W}$ in terms of the genetic variance $V_{\mathrm{G}}$ and of higher cumulants $\left(K_{4}, K_{6}\right)$. Furthermore, these higher cumulants can in turn be expressed in terms of the genetic variance, assuming that $p_{j} q_{j}$ is small at each locus (rare-alleles approximation, e.g. Barton and Turelli, 1987; Turelli and Barton, 1990): one obtains in particular $K_{4} \approx a^{2} V_{\mathrm{G}}[3 m /(2+m)]$, $K_{6} \approx a^{4} V_{\mathrm{G}}[15 m /(4+m)]$, which fits well with the simulation results (see Figure 1 in Supplementary File S3). Finally, the methods developed by Turelli and Barton (1994) can be used to calculate $V_{\mathrm{G}}$ at equilibrium in this non-Gaussian regime. As shown in Supplementary File S3, the result (which has to be obtained numerically) fits well with the simulation results as long as $a_{\mathrm{s}}^{2}$ is not too large, and $Q \geq 2$; however, we could not find any simple analytical expression for $\bar{W}$ in this non-Gaussian regime. From the expressions of $K_{4}$ and $K_{6}$ above, one predicts that the Gaussian regime should be left 
more rapidly (as $a^{2}$ increases) for larger $m$, since $K_{4}$ and $K_{6}$ are increasing functions of $m$; Figure 3 (left) confirms that this is indeed the case. However, Figure 3 right indicates that for a fixed $\bar{s}, \bar{W}$ is relatively insensitive to $m$.

Figure 4 explores the effect of linkage on mean fitness: for $U=0.5$ and $n=50$, the effect of linkage disequilibria on $\bar{W}$ remains small as long as the harmonic mean recombination rate $r_{\mathrm{H}}$ is not too small (with $L=10000$ loci, $r_{\mathrm{H}} \approx 0.053$ for a map length of 1 Morgan, while $r_{\mathrm{H}} \approx 0.3$ for a map length of 10 Morgans - see Appendix B). In this regime, increasing linkage tends to reduce mean fitness (through an increase in genic variance $V_{\mathrm{g}}$ ), and equation 7 matches well with the simulation results (except for $Q=1$ where the fit is less good). For tighter linkage, however (roughly, $R<1$ ) simulations results depart from the prediction from equation 7 (which assumes that the contribution of linkage disequilibria to the genetic variance remains small) and show that $\bar{W}$ increases as recombination decreases: overall, linkage thus has a non-monotonic effect on mean fitness. Interestingly, equation 7 indicates that the contribution of linkage disequilibria becomes more important when the number of traits under selection $(n)$ is small. Figure 5 confirms this result, showing that lower values of $n$ lead to a stronger decrease in $\bar{W}$ for both $\bar{s}=0.01$ and $\bar{s}=0.1$, while the effect is less marked (and may even be reversed) when $\bar{s}=0.001$. This effect of $n$ can be understood as follows: mean fitness decreases when the sum of genetic variances for the different traits $\sum_{i=1}^{n} V_{\mathrm{G}, i}$ increases. Due to the symmetry of our model, the two components of the genetic variance (the genic variance $V_{\mathrm{g}, i}$, and the contribution of linkage disequilibria $d_{i}$, see Appendix A) should be the same for all traits. As shown in Supplementary File S2 (see also Turelli, 1985; Bürger, 2000), when the effects of linkage disequilibria are neglected the genetic variance for each trait at equilibrium is 
proportional to $1 / n$ (roughly, $p_{j} q_{j}$ at each locus scales with $1 / m$, since the strength of selection at each locus increases with $m$; however, a proportion $m / n$ only of loci contribute to trait $i$ : therefore, the effect of $m$ cancels and $V_{\mathrm{g}, i}$ scales with $1 / n$ ). As a consequence $\bar{W}$ (which depends on the product $n V_{\mathrm{G}}$ ) is independent of $n$. The contribution of associations between loci depends on all pairwise linkage disequilibria and is proportional to $V_{\mathrm{g}}{ }^{2}$ (see Supplementary File S2) and therefore to $1 / n^{2}$. As a consequence, the overall contribution of linkage disequilibria to mean fitness is proportional to $1 / n$. However, pairwise linkage disequilibria may not be the only cause of the strong reduction of $\bar{W}$ shown on Figure 5 at low values of $n$ (for $\bar{s}=0.01$ and 0.1 ): indeed, simulations indicate that for these low values of $n$, distributions of phenotypic traits in the population depart from Gaussian distributions (fourth and sixth cumulants become significantly different from zero), in which case the results derived in Supplementary File S2 (which assume Gaussian distributions of phenotypic traits) do not hold.

Effects of population size and number of loci. Many of the results shown above assume a large population size and large number of loci. Figure 6 explores the effects of varying population size: in the case of a sexual population (Figure 6A), smaller population sizes tend to decrease $\bar{W}$ when the average fitness effect of mutations is sufficiently strong (right part of the figure). However, for lower values of $\bar{s}$ one observes the opposite effect, with higher values of mean fitness for lower population sizes. With a Gaussian fitness function $(Q=2$, as in Figure 6), a diffusion model can be used to predict the equilibrium genetic variance under selection, mutation and drift, assuming that mean phenotypes stay at the optimum (Bulmer, 1972). In Supplementary File S4, 
we obtain an approximation for mean fitness (in terms of hypergeometric functions) valid for $N u \ll 1$, which fits well with simulation results for $N=500$ (dotted curve in Figure 6A). In contrast to the sexual case, the effects of population size are less marked in the case of asexual organisms (Figure $6 \mathrm{~B}$ ), and $\bar{W}$ always decreases as $N$ decreases.

These effects of finite population size can be understood as follows. Genetic drift affects mean fitness through two different effects: an effect on the genetic variance maintained at equilibrium, which is usually (but not always) lower in smaller populations, and an effect on mean phenotypes, which may be displaced from the optimum due to drift. In the case of a Gaussian fitness function $(Q=2)$, these two effects can easily be separated: indeed, log-fitness equals $-\sum_{i=1}^{n}\left(z_{i}-z_{i}^{*}\right)^{2} /\left(2 V_{\mathrm{S}}\right)$, where $z_{i}$ is the value of phenotype $i$ and $z_{i}^{*}$ the optimal value for this phenotype (fixed to zero in our model). Therefore, the average log-fitness is given by $-\sum_{i=1}^{n}\left(V_{\mathrm{G}, i}+\left(\overline{z_{i}}-z_{i}^{*}\right)^{2}\right) /\left(2 V_{\mathrm{S}}\right)$, where $V_{\mathrm{G}, i}$ is the genetic variance for trait $i$ and $\overline{z_{i}}$ the average value of trait $i$ (see also Charlesworth, 2013). Assuming that $\overline{\ln W} \approx \ln \bar{W}$, mean fitness can thus be written as a product of two terms:

$$
\bar{W} \approx \exp \left[-\frac{\sum_{i=1}^{n} V_{\mathrm{G}, i}}{2 V_{\mathrm{S}}}\right] \exp \left[-\frac{\sum_{i=1}^{n}\left(\overline{z_{i}}-z_{i}^{*}\right)^{2}}{2 V_{\mathrm{S}}}\right] .
$$

The first term (denoted hereafter $\bar{W}_{V_{\mathrm{G}}}$ ) shows that increasing the variance of phenotypic traits tends to decrease mean fitness, while the second term (denoted hereafter $\left.\bar{W}_{\bar{z}}\right)$ shows that displacing mean phenotypes from the optimum also decreases $\bar{W}$. Simulations show that injecting equilibrium values of genetic variances $V_{\mathrm{G}, i}$ and mean phenotypes $\overline{z_{i}}$ (measured in the simulations) into equation 9 accurately predict $\bar{W}$ for all parameters tried with $Q=2$, as long as $\log _{10}(\bar{s}) \leq-1$ (results not shown). Note that all the mathematical derivations performed in the different Supplementary Files 
assume that mean phenotypes are at the optimum, and therefore that $\bar{W}_{\bar{z}}=1$. Supplementary Figure S2 shows measures of $\bar{W}_{V_{\mathrm{G}}}$ and $\bar{W}_{\bar{z}}$ for the different values of $N$ and $\bar{s}$ explored in Figure 6 . These results can be summarized as follows: in a sexual population (Figure $6 \mathrm{~A}$ ), $\bar{W}_{\bar{z}}$ stays close to 1 in most cases, but may be slightly less than 1 (around $0.97-0.98$ ) for $N=500$ : mean phenotypes thus generally stay close to their optimal values, and the mutation load is mainly generated by the term $\bar{W}_{V_{\mathrm{G}}}$. When mutations tend to have strong fitness effects $\left(\log _{10}(\bar{s}) \geq-2\right)$, drift tends to increase genetic variances $V_{\mathrm{G}, i}$ (by increasing $\overline{p_{j} q_{j}}$ at each locus $j$ above its value at mutation-selection balance), and thus decreases $\bar{W}_{V_{\mathrm{G}}}$ - note that this effect is taken into account by the diffusion model (equation 6 in Supplementary File S4). When mutations tend to have weak fitness effects $\left(\log _{10}(\bar{s}) \leq-3\right)$ however, drift tends to reduce $V_{\mathrm{G}, i}$ by bringing the different loci closer to fixation, thereby increasing $\bar{W}_{V_{\mathrm{G}}}$ (and hence $\bar{W}$ ). By contrast, in an asexual population drift always reduce the genetic variance $\left(\bar{W}_{V_{\mathrm{G}}}\right.$ increases as $N$ decreases), but also has a substantial effect on mean phenotypes ( $\bar{W}_{\bar{z}}$ decreases as $N$ decreases). Because the second effect is stronger than the first, the overall effect of drift is to reduce the mean fitness of asexuals.

Our model is somehow artificial, however, as we assumed that the fitness optimum is located at the origin $\left(z_{i}^{*}=0\right.$ for all $\left.i\right)$. Due to the symmetry of the model, one expects that mean phenotypes should be at the origin (and thus at the optimum) in the regime where dynamics are mainly driven by mutation and drift (low $\bar{s})$, when the number of loci is large: for example if a large, random proportion of loci are fixed for allele 1 and the other loci fixed for allele 0, mean phenotypes should be close to zero since the sum of all mutational effects (corresponding to the effects of fixed alleles 1) converges to the average effect of mutations, which is zero. It seems artificial, however, 
to assume that optimal trait values are precisely the values towards which the population should converge at mutation-drift equilibrium, and it seems more likely that in many cases, mean phenotypes at mutation-drift equilibrium should be far from the optimal values (decreasing the value of $\bar{W}_{\bar{z}}$ in the mutation-drift regime). In order to explore that, we modified our simulation program so that the fitness optimum is not located at the origin of the phenotypic space $\left(z_{i}=0\right.$ for all $\left.i\right)$. To do this, we select a genotype sufficiently distant from the origin (in phenotypic space) so that when setting optimal phenotypic values $z_{i}^{*}$ to the values coded by this genotype, fitness at the origin is less than 0.1. At the start of the simulation, the population is fixed for the genotype corresponding to the fitness optimum. At mutation-drift balance, the population is still expected to converge to the origin (due to the symmetry of the distribution of mutational effects), where fitness is lower than 0.1. Results are shown on Figures 6C and $6 \mathrm{D}$ in the case of a sexual and asexual population, respectively. As can be seen on these Figures, the position of the fitness optimum has little effect as long as selection is sufficiently strong $\left(\log _{10}(\bar{s}) \geq-3\right)$, since in this case selection maintains the population near the optimum. For lower $\bar{s}$, however, mutation and drift tend to bring populations away from the optimum (and closer to the origin), which decreases $\bar{W}_{\bar{z}}$ and thus decreases mean fitness (see also Supplementary Figure S2), this effect being more pronounced in asexual than in sexual populations (in sexuals, this effect becomes important only for $\bar{s}=10^{-5}$ ). Note that we could not obtain results for $\bar{s}=10^{-6}$, because with such low mutational effects (and with 10000 loci) it was not possible to find a genotype sufficiently distant from the origin so that fitness at the origin is lower than 0.1 when setting the optimum to the phenotypic values coded by this genotype. Interestingly, in the sexual case (Figure 6C) one still observes an intermediate range 
of $\bar{s}\left(\bar{s}=10^{-4}, 10^{-3}\right)$ where decreasing population size increases mean fitness: in this regime, drift tends to bring each locus closer to fixation (thus reducing $V_{\mathrm{G}, \mathrm{i}}$ ), but selection still maintains mean phenotypes $\overline{z_{i}}$ close to the optimum. Decreasing $\bar{s}$ (or decreasing $N$ further, as shown in Supplementary Figure S2 for $N=100$ ) generates departures of mean phenotypes from the optimum, decreasing $\bar{W}_{\bar{z}}$ (and thus $\bar{W}$ ). Finally, Supplementary Figure S3 shows that changing the number of loci $L$ (for a fixed $U$ ) has generally stronger effects in the case of sexual populations, where in the low $\bar{s}$ regime, decreasing the number of loci decreases the genetic variance (and thus increases $\bar{W})$.

(1)

\section{DISCUSSION}

As we have seen in introduction, different forms of models have been proposed to predict the overall effect of recurrent mutation on the mean fitness of populations. Typically, models representing deleterious mutations (without including a phenotypic dimension) do not consider possible compensatory effects among mutations, and assume that epistasis is the same for all pairs of mutations (Kimura and Maruyama, 1966; Charlesworth, 1990). Using this type of model, Kimura and Maruyama (1966) predicted that epistasis should have no effect on the mean fitness of asexuals (which should remain close to $e^{-U}$ ), while it should increase the fitness of sexuals if it is negative, and decrease $\bar{W}$ if it is positive. On the other hand, models representing stabilizing selection on a set of quantitative phenotypic traits (such as Fisher's geometric model) provide a natural way of incorporating compensatory effects among mutations and distributions of selection coefficients and epistatic effects. In this paper, we used a 
general model that allows us to play with the number of traits under selection $(n)$, the number of traits affected by a given mutation $(m)$ and the shape of the fitness peak $(Q)$. Interestingly, this last parameter was already shown by others to affect the average sign of epistasis among pairs of mutations: epistasis is positive on average when the fitness peak is sharper than a Gaussian function $(Q<2)$, while it is negative when the fitness peak is flatter than a Gaussian $(Q>2)$, and equal to zero when fitness is exactly Gaussian (Martin et al., 2007; Gros et al., 2009). Furthermore, the average deleterious effect of mutations at the optimum $\bar{s}$ can be expressed as a simple function of the variance of scaled phenotypic effects of mutations $a_{\mathrm{s}}^{2}$ and the parameters $m$ and $Q$ (equation 3).

In the case of an asexual population, we found that mean fitness stays indeed close to $e^{-U}$ when mutations tend to have strong fitness effects; however, $\bar{W}>e^{-U}$ under weaker fitness effects of mutations (Gaussian regime), due to the possibility of compensatory effects among mutations at different loci. For a fixed $\bar{s}$, mean fitness is higher under positive epistasis $(Q<2)$ than under negative epistasis $(Q>2)$; this is probably due to the fact that selection for compensatory mutations is stronger when the fitness peak is sharp, keeping the population closer to the fitness optimum. In contrast, under negative epistasis the fitness landscape presents a plateau around the optimum, and at equilibrium many genotypes are located near the edge of this plateau, where the effect of deleterious mutations tends to be strong. Finally, we showed that under this regime mean fitness can be expressed as a simple function of $U, \bar{s}, n$ and $Q$ (equation 5), which indicates that $\bar{W}$ decreases as the number of traits under selection $n$ increases.

In a sexual population, mean fitness is also at $e^{-U}$ when mutations have very 
strong fitness effects: this is expected, as mutations stay at very low frequency and epistatic interactions among mutations have thus little effect. For weaker mutational effects, Kimura and Maruyama (1966)'s prediction is verified: positive epistasis decreases $\bar{W}$, while negative epistasis increases it; furthermore, we showed that $\bar{W}$ converges to a very simple expression $\left(e^{-\frac{2 U}{Q}}\right)$ when the phenotypic effect of mutations is sufficiently weak, so that distributions of phenotypic values in the population are nearly Gaussian. Although this expression assumes that linkage disequilibria among loci can be neglected, effects of genetic associations can be computed, showing that linkage disequilibria tend to reduce $\bar{W}$ as long as they remain weak, the importance of this effect increasing as the number of selected traits $n$ decreases. Contrarily to results obtained previously assuming $N U \ll 1$ (Hartl and Taubes, 1998; Poon and Otto, 2000; Tenaillon et al., 2007; Lourenço et al., 2011), our model predicts a higher mean fitness when the number of traits is large, through this effect on linkage disequilibrium. Figure 5 indeed shows important reductions in fitness when the number of traits decreases (for moderate to strong fitness effects of mutations, of the order 0.01 to 0.1 ), which may also involve higher-order genetic associations (since distributions of phenotypic values depart from Gaussian distributions for very low values of $n$ ). Rather than the "cost of complexity" often described at low mutation rate, we thus predict a "cost of simplicity" when the overall mutation rate is large (as shown by equation 7 , this effect is proportional to $U^{2}$ and should thus become negligible when the overall mutation rate is small). Note that this cost does not depend on the degree of pleiotropy $(m)$ of individual mutations, but only on the total number of traits under selection. Interestingly, these results do not depend on the precise shape of the distribution of mutational effects (which was Gaussian in the simulations), since the derivation of equations 6 and 
7 in Supplementary File S2 makes no assumption on this distribution. It does assume uncorrelated mutational effects on the different traits, however, and it would be interesting to extend our analysis by incorporating mutational and/or selective correlations among traits - as shown by Martin and Lenormand (2006), the overall effect of such correlations is to reduce the effective number of phenotypic traits (see also Zhang and Hill, 2003 for an analysis of the effects of correlations among traits). Furthermore, different ways of incorporating restricted pleiotropy could be considered: in particular, Welch and Waxman (2003) proposed a model in which different sets of traits are affected by different subsets of loci (modular pleiotropy), and it would be interesting to explore how this form of modularity would affect our results.

Regarding genetic architecture, an important assumption of our model is the fact that loci are biallelic. By contrast, Lande (1980)'s analysis considered an infinite number of alleles per locus (continuum-of-alleles model); in that case, assuming diagonal mutation and selection matrices (no mutational or selective covariance among traits) and neglecting linkage disequilibria, one obtains that the mean log-fitness should be $-\sqrt{\frac{n}{2} L U \bar{s}}$ (where $L$ is the number of loci) in the case of a Gaussian fitness function (this stems from the fact that the contribution of each locus to the genetic variance is $\sqrt{u a^{2} V_{\mathrm{S}}}$, where $u$ is the per-locus mutation rate). This contrasts with the result that mean log-fitness should be $\approx-U$ when $Q=2$ in biallelic models, and indicates that assumptions about the number of alleles per locus may have important effects. However, a key assumption of Lande's analysis is the fact that distributions of allelic effects in the population are Gaussian at each locus, which is much stronger than the assumption that distributions of overall phenotypes are Gaussian, and may not hold for realistic values of per-locus mutation rates and mutational variances (e.g., Turelli, 
1984). It would thus be interesting to explore how sensitive are our results to assumptions regarding the number of alleles per locus. In particular, our assumption of biallelic loci should not hold when $N u \sim 1$ (in which case many alleles may segregate at the same locus).

Finally, we found that the degree of pleiotropy of mutations $(m)$ affects mean fitness mostly through its effect on $\bar{s}$, in regimes where $\bar{W}$ is affected by $\bar{s}$ : in particular the Gaussian regime in asexual populations (equation 5), and the regime where selection at each locus is weaker than mutation and drift in sexual populations (equation 8). This contrasts with the result obtained by Lourenço et al. (2011) in the low mutation limit $(N U \ll 1)$, showing that the average fitness at equilibrium depends on the total number of selected traits but not on the degree of mutational pleiotropy. Another difference concerns the effect of population size: although the load always increases as population size decreases when $N U \ll 1$ (Hartl and Taubes, 1998; Poon and Otto, 2000; Tenaillon et al., 2007; Lourenço et al., 2011), we found that in sexuals, the mutation load may actually be lower at smaller population sizes in the weak selection regime (Figure 6). As we have seen, this occurs whenever the fitness effect of mutations is small $\left(\bar{s}=10^{-4}, 10^{-3}\right.$ in the case of Figure 6$)$, so that diversity at each locus $\left(p_{j} q_{j}\right)$ at mutation-selection balance is substantial, in which case drift tends to reduce this diversity and therefore reduces the genetic variance $V_{\mathrm{G}}$ - the same effect has also recently been discussed by Charlesworth (2013) in a 1-trait model. For very low fitness effects of mutations, however, mutation and drift may displace mean phenotypes away from the optimum and substantially reduce mean fitness (Figures 6C and D). This last effect may only occur for a restricted range of parameters, since when $\bar{s}$ becomes very low, very large numbers of loci are required for fitness to decrease 
significantly (see also Charlesworth, 2013).

In general, we found that asexual populations have a higher mean fitness than sexual populations, in agreement with previous models of stabilizing selection with a constant optimum (e.g., Charlesworth, 1993): this is due to the fact that genotypes coding for optimal trait values are preserved by asexual reproduction, but broken by recombination. Nevertheless, sexuals may have a higher mean fitness than asexuals when epistasis among mutations is negative on average $(Q>2)$, as long as $\bar{s}$ is not too small (so that $\bar{W}$ stays close to $e^{-U}$ in asexuals, but reach higher values in sexuals); this occurs for example for $Q=6$, when $\bar{s} \geq 10^{-4}$ (for the parameter values used in Figures 1 and 2). Sexuals may also have a higher mean fitness when the effects of drift are important: in particular, Figure $6 \mathrm{C}$ and $6 \mathrm{D}$ show that deviations of mean phenotypes from their optimal values caused by drift are generally more important in asexual than in sexual populations (for low $\bar{s}$ ). Although we only observed this last effect for restricted ranges of parameters, it may become more important in the case of populations subdivided into many small demes. Previous models explored the evolution of recombination modifiers under stabilizing selection (Charlesworth, 1993; Barton, 1995), but ignored the effects of genetic drift: it would thus be interesting to extend these models to the case of finite (or spatially structured) populations. Exploring the effects of diploidy and of the mating system would be other possible extensions of the present work.

Acknowledgements. We thank Nick Barton, Harold de Vladar, Pierre-Alexis Gros, Guillaume Martin, Sylvain Glémin and two anonymous reviewers for helpful discussions and comments, and the bioinformatics and computing service of Roscoff's Bio- 
596 logical Station for computing time. This work was supported by the French Agence ${ }_{597}$ Nationale de la Recherche (ANR-11-BSV7-013-03). 
$\operatorname{Var}\left[z_{i}\right]$. Because we assume additive effects of the different loci on phenotypic traits ${ }_{601}\left(z_{i}=\sum_{j} r_{i j} X_{j}\right.$, where the sum is over all loci $\left.j\right), V_{\mathrm{G}, i}$ can be decomposed into two ${ }_{602}$ terms (e.g., Lynch and Walsh, 1998): $V_{\mathrm{G}, i}=V_{\mathrm{g}, i}+d_{i}$, where $V_{\mathrm{g}, i}=\sum_{j} r_{i j}^{2} p_{j}\left(1-p_{j}\right)$ ${ }_{603}$ is the genic variance $\left(p_{j}\right.$ being the frequency of allele 1 at locus $j$ in the population), ${ }_{604}$ and $d_{i}=\sum_{j \neq k} r_{i j} r_{i k} D_{j k}$ the effect of linkage disequilibria on the genetic variance ${ }_{605}\left(D_{j k}=\operatorname{Cov}\left[X_{j}, X_{k}\right]\right.$ being the linkage disequilibrium between loci $j$ and $\left.k\right)$. In the 606 case of an asexual population, the genetic variance at equilibrium can be calculated 607 by considering the whole genome as a single locus with a large number of alleles ${ }_{608}$ (Supplementary File $\mathrm{S} 1$ ). In the sexual case, $V_{\mathrm{g}, i}$ and $d_{i}$ at equilibrium can be computed 609 using the methods developed by Turelli and Barton (1990), extended to deal with ${ }_{610}$ multiple traits under selection (Supplementary File S2). Due to the symmetry of the ${ }_{611}$ model we expect that $V_{\mathrm{G}, i}$ and $V_{\mathrm{g}, i}$ should be the same for all traits at equilibrium, ${ }_{612}$ and are thus simply denoted $V_{\mathrm{G}}$ and $V_{\mathrm{g}}$ in the text. 

rate among pairs of loci $r_{\mathrm{H}}$ (that appears in equation 7 ) can be computed as follows. ${ }_{617}$ the distance between two loci separated by $i$ between-locus intervals is $i R /(L-1)$. ${ }_{618}$ Furthermore, the number of different pairs of loci separated by $i$ intervals is $L-i$. ${ }_{619}$ Finally, the rate of recombination between two loci at genetic distance $x$ (probability 620 that an odd number of cross-overs occurs within the interval) is given by Haldane's ${ }_{621}$ mapping function: $r(x)=\frac{1}{2}\left(1-e^{-2 x}\right)$, yielding:

$$
\frac{1}{r_{\mathrm{H}}}=\frac{1}{\frac{1}{2} L(L-1)} \sum_{i=1}^{L-1} \frac{L-i}{\frac{1}{2}\left[1-\exp \left(-2 i \frac{R}{L-1}\right)\right]}
$$

${ }_{622}$ which has to be evaluated numerically. For $L=10000$ and $R=10$, one obtains ${ }_{623} r_{\mathrm{H}} \approx 0.3$ 
Barton, N. H. 1986. The maintenance of polygenic variation through a balance between mutation and stabilizing selection. Genet. Res. 47:209-216.

. 1989. The divergence of a polygenic system subject to stabilizing selection, mutation and drift. Genet. Res. 54:59-77.

Barton, N. H. and M. Turelli. 1987. Adaptive landscapes, genetic distance and the evolution of quantitative characters. Genet Res 49:157-173.

Bulmer, M. G. 1971. The effect of selection on genetic variability. Am. Nat. 105:201211.

- 1972. The genetic variability of polygenic characters under optimizing selection, mutation and drift. Genet. Res. 19:17-25.

- 1974. Linkage disequilibrium and genetic variability. Genet. Res. 23:281-289.

- 1985. The Mathematical Theory of Quantitative Genetics, 2nd edition. Oxford University Press, Oxford.

- 1989. Maintenance of genetic variability by mutation-selection balance: a child's guide through the jungle. Genome 31:761-767.

Bürger, R. 2000. The Mathematical Theory of Selection, Recombination, and Mutation. Wiley, Chichester, U.K. 
${ }_{644}$ Charlesworth, B. 1990. Mutation-selection balance and the evolutionary advantage of ${ }_{645} \quad$ sex and recombination. Genet. Res. 55:199-221.

646

647

648

649

650

651

652

653

654

- 1993. Directional selection and the evolution of sex and recombination. Genet. Res. 61:205-224.

- 2013. Why we are not dead 100 times over. Evolution in press.

Charlesworth, B. and D. Charlesworth. 1999. The genetic basis of inbreeding depression. Genet. Res. 74:329-40.

Charlesworth, D. 2006. Evolution of plant breeding systems. Curr. Biol. 16:R726R735.

Crow, J. F. 1970. Genetic loads and the cost of natural selection. Pp. 128-177 in K. Kojima, ed. Mathematical Topics in Population Genetics. Springer-Verlag, Berlin.

Fisher, R. A. 1930. The genetical theory of natural selection. Clarendon Press, Oxford.

Gros, P.-A., H. Le Nagard, and O. Tenaillon. 2009. The evolution of epistasis and its links with genetic robustness, complexity and drift in a phenotypic model of adaptation. Genetics 182:277-293.

Haldane, J. B. S. 1932. The causes of evolution. Longmans, Green, London.

Hartl, D. L. and C. H. Taubes. 1998. Towards a theory of evolutionary adaptation. Genetica 103:525-33.

Hastings, W. K. 1970. Monte Carlo sampling methods using Markov chains and their applications. Biometrika 57:97-109. 
Johnson, T. and N. H. Barton. 2005. Theoretical models of selection and mutation on quantitative traits. Phil. Trans. Roy. Soc. (Lond.) B 360:1411-1425.

Keightley, P. D. and S. P. Otto. 2006. Interference among deleterious mutations favours sex and recombination in finite populations. Nature 443:89-92.

Kimura, M. 1965. A stochastic model concerning the maintenance of genetic variability in quantitative characters. Proc. Natl. Acad. Sci. U. S. A. 54:731-736.

Kimura, M. and T. Maruyama. 1966. The mutational load with epistatic gene interactions in fitness. Genetics 54:1337-1351.

Kondrashov, A. S. 1988. Deleterious mutations and the evolution of sexual reproduction. Nature 336:435-440.

Lande, R. 1976. The maintenance of genetic variability by mutation in a polygenic character with linked loci. Genet. Res. 26:221-235.

- 1977. The influence of the mating system on the maintenance of genetic variability in polygenic characters. Genetics 86:485-498.

— 1980. The genetic covariance between characters maintained by pleiotropic mutations. Genetics 94:203-215.

Lande, R. and D. W. Schemske. 1985. The evolution of self-fertilization and inbreeding depression in plants. I. Genetic models. Evolution 39:24-40.

Lourenço, J., N. Galtier, and S. Glémin. 2011. Complexity, pleiotropy and the fitness effect of mutations. Evolution 65:1559-1571. 
696

Lynch, M. and J. B. Walsh. 1998. Genetics and Analysis of Quantitative Traits. Sinauer Associates, Sunderland, MA.

Martin, G., S. F. Elena, and T. Lenormand. 2007. Distributions of epistasis in microbes fit predictions from a fitness landscape model. Nat. Genet. 39:555-560.

Martin, G. and T. Lenormand. 2006. A general multivariate extension of Fisher's geometrical model and the distribution of mutation fitness effects across species. Evolution 60:893-907.

Orr, H. A. 1998. The population genetics of adaptation: the distribution of factors fixed during adaptive evolution. Evolution 52:935-949.

- 2000. Adaptation and the cost of complexity. Evolution 54:13-20.

Otto, S. P. 2009. The evolutionary enigma of sex. Am. Nat. 174:S1-S14.

Otto, S. P. and D. B. Goldstein. 1992. Recombination and the evolution of diploidy. Genetics 131:745-751.

Otto, S. P. and J. C. Marks. 1996. Mating systems and the evolutionary transition between haploidy and diploidy. Biol. J. Linn. Soc. 57:197-218.

Poon, A. and S. P. Otto. 2000. Compensating for our load of mutations: freezing the meltdown of small populations. Evolution 54:1467-1479.

Rowe, L. and D. Houle. 1996. The lek paradox and the capture of genetic variance by condition dependent traits. Proc. Roy. Soc. (Lond.) B 263:1415-1421.

Sella, G. 2009. An exact steady-state solution of Fisher's geometric model and other models. Theor. Popul. Biol. 75:30-34. 
Sella, G. and A. E. Hirsh. 2005. The application of statistical physics to evolutionary biology. Proc. Natl. Acad. Sci. U. S. A. 102:9541-9546.

Tenaillon, O., O. K. Silander, J.-P. Uzan, and L. Chao. 2007. Quantifying organismal complexity using a population genetic approach. PLoS One 2:e217.

Turelli, M. 1984. Heritable genetic variation via mutation-selection balance: Lerch's zeta meets the abdominal bristle. Theor. Popul. Biol. 25:138-193.

- 1985. Effects of pleiotropy on predictions concerning mutation-selection balance for polygenic traits. Genetics 11:165-195.

Turelli, M. and N. H. Barton. 1990. Dynamics of polygenic characters under selection. Theor. Popul. Biol. 38:1-57.

- 1994. Genetic and statistical analyses of strong selection on polygenic traits: what, me normal? Genetics 138:913-941.

Waxman, D. and J. R. Peck. 1998. Pleiotropy and the preservation of perfection. Science 279:1210-1213.

Welch, J. J. and D. Waxman. 2003. Modularity and the cost of complexity. Evolution $57: 1723-1734$.

Wilke, C. O. and C. Adami. 2001. Interaction between directional epistasis and average mutational effects. Proc. Roy. Soc. (Lond.) B 268:1469-1474.

Wright, S. 1935. Evolution in populations in approximate equilibrium. J. Genet. $30: 257-266$. 
725

726

727

728

729

730

Zhang, X.-S. 2012. Fisher's geometrical model of fitness landscape and variance in fitness within a changing environment. Evolution 66:2350-2368.

Zhang, X.-S. and W. G. Hill. 2003. Multivariate stabilizing selection and pleiotropy in the maintenance of quantitative genetic variation. Evolution 57:1761-1775.

- 2005. Genetic variability under mutation selection balance. Trends Ecol. Evol. 20:468-470. 

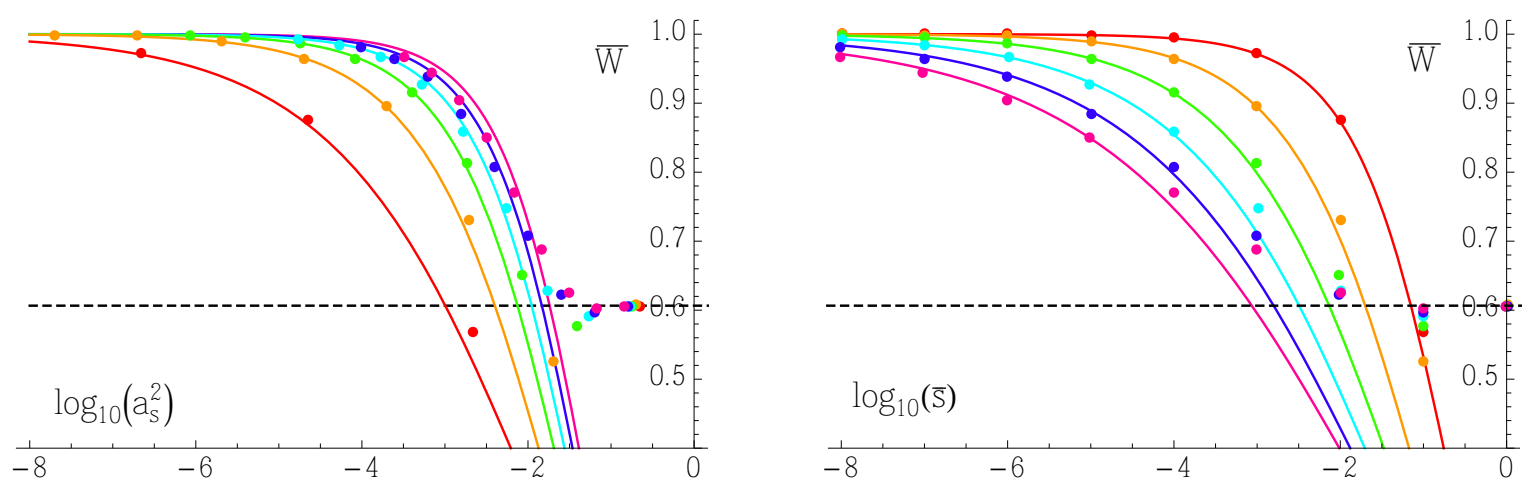

Figure 1. Mean fitness of an asexual population as a function of $a_{\mathrm{s}}^{2}$ and $\bar{s}$. X-axes correspond to the mutational variance on scaled traits $a_{\mathrm{s}}^{2}=a^{2} /\left(2 V_{\mathrm{S}}\right)^{\frac{2}{Q}}$ (left) and the average deleterious effect of mutations (on log-fitness) at the optimum $\bar{s}$ (right). The different colors correspond to different shapes of the fitness function, controlled by the parameter $Q: Q=1,2,3,4,5,6$ from red to purple. The dashed horizontal line corresponds to $e^{-U}$, while colored curves are predictions from equation 5 , and dots are simulation results. Parameter values are $U=0.5, n=50, m=5$; in the simulations $V_{\mathrm{S}}=0.5$, population size is set to $N=50000$ and the number of loci to $L=10000$. 

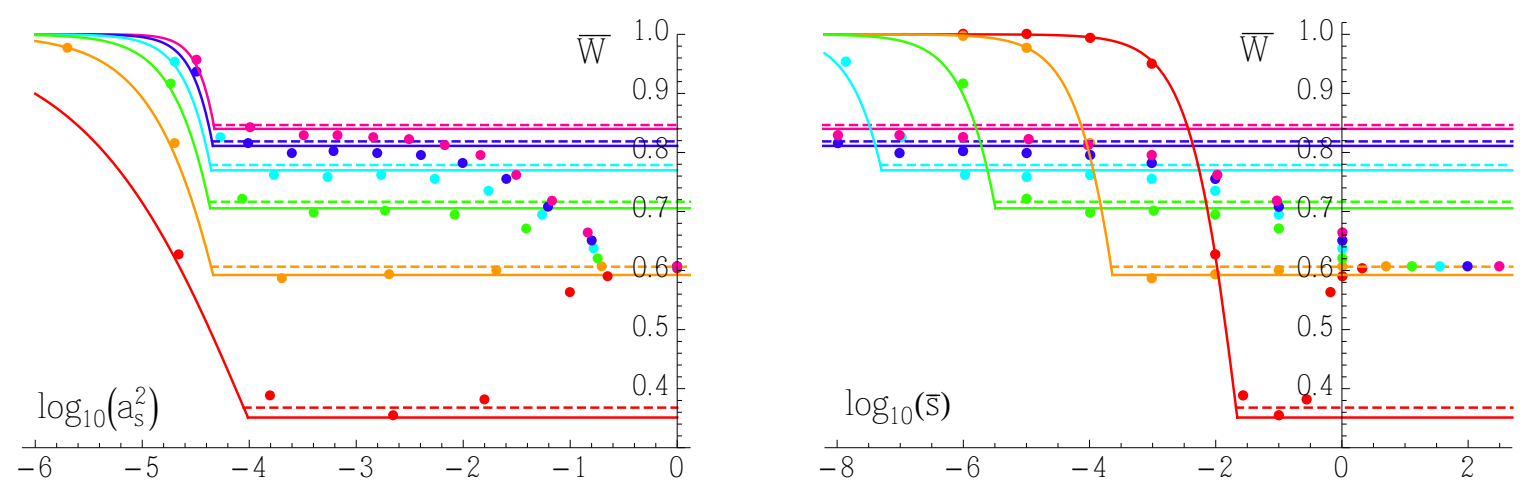

Figure 2. Mean fitness of a sexual population as a function of $a_{\mathrm{s}}^{2}$ and $\bar{s}$ (defined as the average effect of mutations on log-fitness at the optimum). Parameter values are the same as in Figure 1, with genome map length $R=10$ Morgans (which leads to $r_{\mathrm{H}} \approx 0.3$, see Appendix B). Dots correspond to simulation results, and curves to different approximations obtained assuming Gaussian distributions of phenotypic traits in the population: equations 6 (dashed lines), 7 (horizontal solid lines) and 8 (solid curves on the left). Note that in the left figure all points are superposed for $\log _{10}\left(a_{\mathrm{s}}^{2}\right)=0$. 

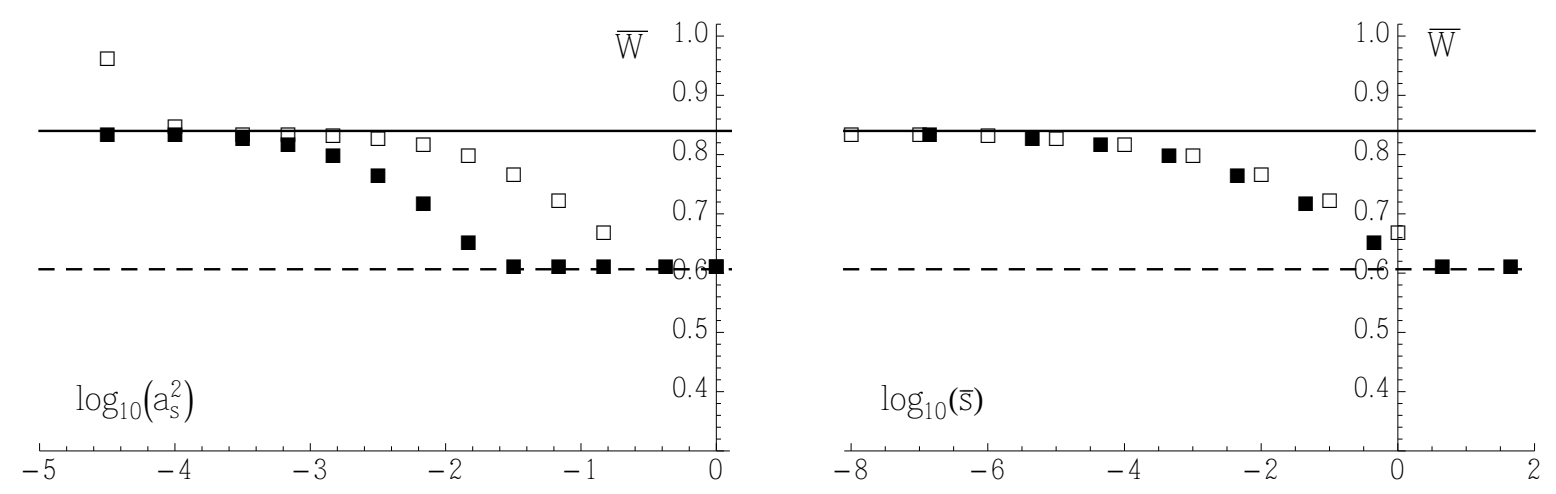

Figure 3. Mean fitness of a sexual population: effect of the degree of pleiotropy $m$. Squares: simulation results for $Q=6, m=5$ (white) and $m=50$ (black); other parameters are as in Figure 2. The solid line corresponds to the prediction from equation 7 (Gaussian regime), and the dashed line to $e^{-U}$. 


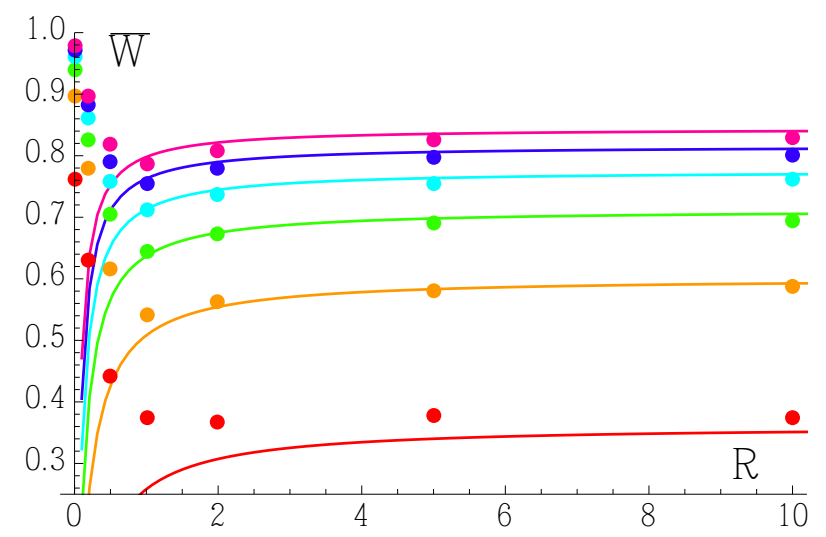

Figure 4. Mean fitness as a function of map length $R$ in Morgans. $R$ corresponds to the mean number of cross-overs per meiosis within the genome; note that $R=0$ corresponds to asexual reproduction. Parameter values are the same as in Figures 1 and 2 , with $a^{2}=0.0002$ in the simulations (so that $\bar{s}=0.001$ for $Q=2$ ). Dots correspond to simulation results, and curves to the prediction from equation 7 . 

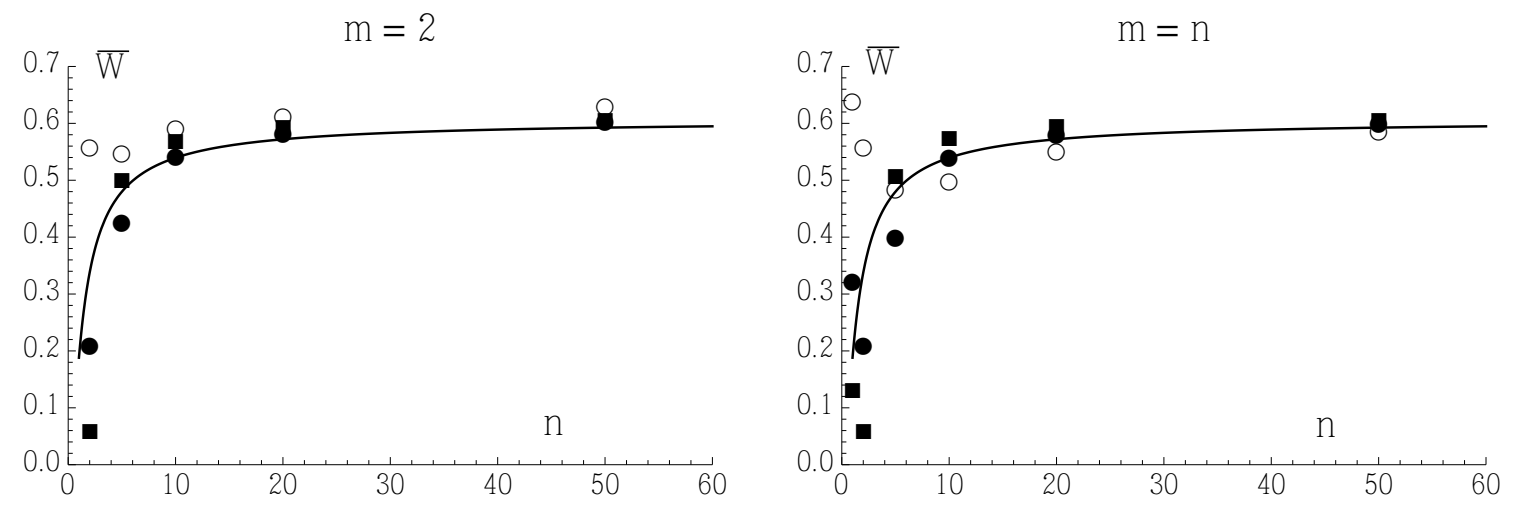

Figure 5. Mean fitness of a sexual population as a function of the number selected traits $n$. The fitness function is Gaussian $(Q=2)$. Curve: prediction from equation 7; dots: simulation results with $\bar{s}=0.001$ (empty circles), $\bar{s}=0.01$ (filled circles) and $\bar{s}=0.1$ (filled squares). Other parameters are as in Figure 2. 
(A)

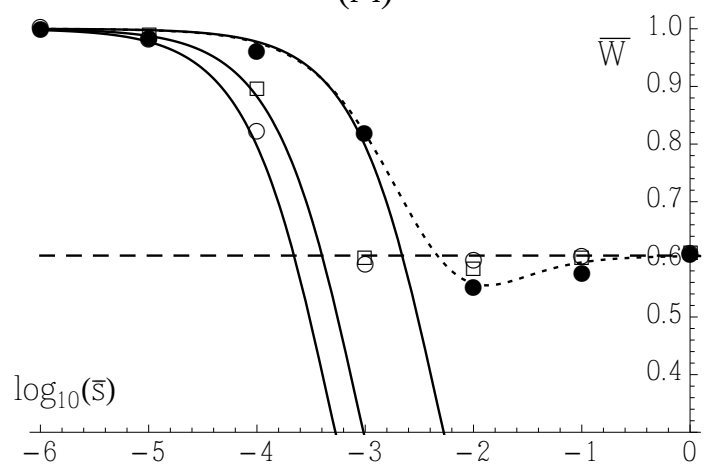

(C)

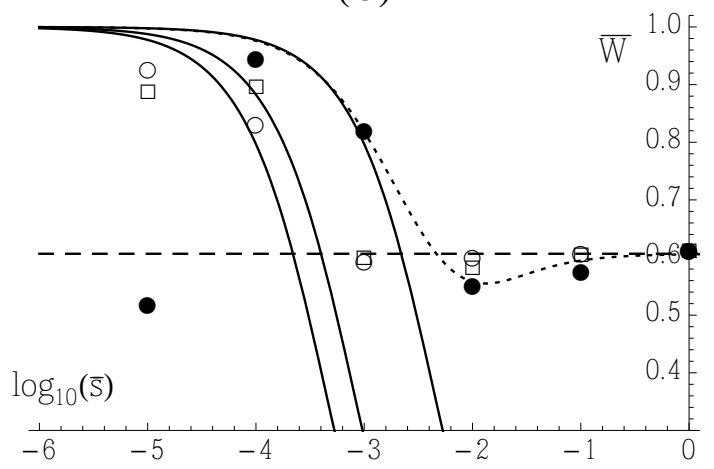

(B)

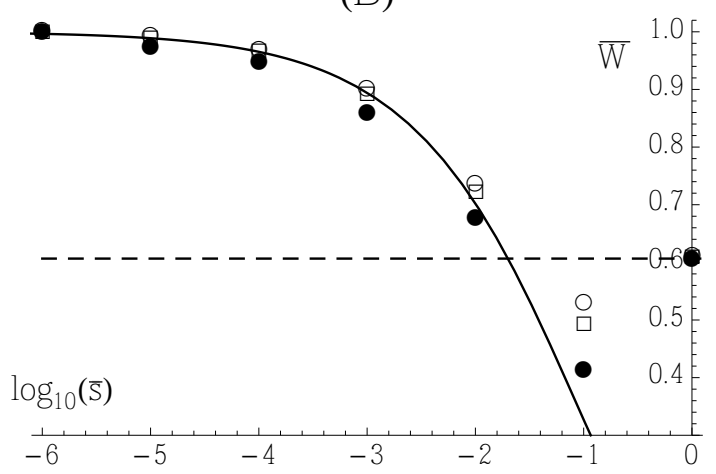

(D)

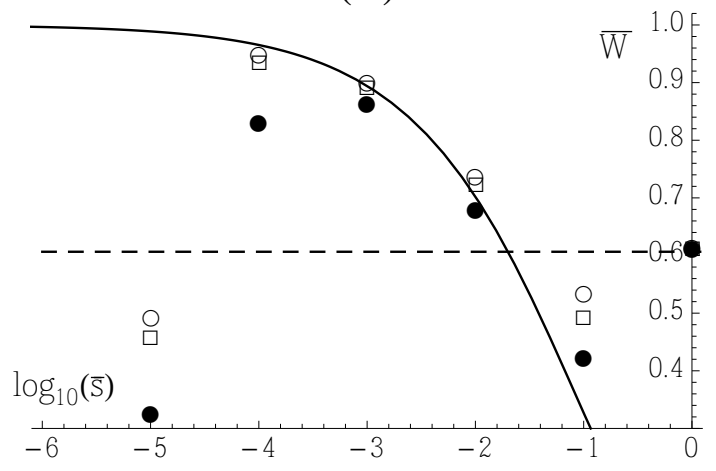

Figure 6. Mean fitness of a sexual (A, C) and asexual (B, D) populations: effects of population size. Dots correspond to simulation results for to $N=50000$ (empty circles), 5000 (empty squares), 500 (filled circles). Other parameters are as in Figure 1 and 2, with $Q=2$. A, B: the optimum is located at the origin (in phenotypic space), that is, in $z_{i}=0$ for all traits $i$, C, D: the optimum is located away from the origin, so that the fitness of an individual at the origin is less than 0.1 (see text for more explanations). Solid curves on the left figures (sexual populations) correspond to the predictions from equation 8 for $N=50000,5000$ and 500 from left to right, while the dotted curves corresponds to equation 6 in Supplementary File S4 (for $N=500$ ). Curves on the right figures (asexual populations) correspond to equation 5. Finally, the dashed horizontal lines correspond to $e^{-U}$. 
Table 1: Parameters and variables of the model.

\begin{tabular}{|c|c|}
\hline$n$ & number of phenotypic traits under selection \\
\hline$m$ & number of traits affected by a single mutation \\
\hline$Q$ & shape of the fitness peak (see equation 1 ) \\
\hline$V_{\mathrm{S}}$ & strength of selection (see equation 1 ) \\
\hline$a^{2}$ & variance of phenotypic effects of mutations \\
\hline$a_{\mathrm{s}}^{2}=a^{2} /\left(2 V_{\mathrm{S}}\right)^{\frac{2}{Q}}$ & variance of mutational effects on scaled traits \\
\hline$L$ & number of loci affecting selected traits \\
\hline$U$ & total rate of mutation per generation on selected loci \\
\hline$u=U / L$ & mutation rate per locus \\
\hline $\bar{s}$ & $\begin{array}{l}\text { mean deleterious effect of mutations (on log-fitness) at the } \\
\text { fitness optimum (given by equation } 3 \text { ) }\end{array}$ \\
\hline$R$ & genome map length (in Morgans) \\
\hline$r_{\mathrm{H}}$ & harmonic mean recombination rate among pairs of loci \\
\hline$N$ & population size \\
\hline$z_{i}$ & value of trait $i$ in a given individual \\
\hline$r_{i j}$ & effect of mutation at locus $j$ on trait $i$ \\
\hline$p_{j}$ & frequency of allele 1 at locus $j$ in the population \\
\hline$D_{j k}$ & linkage disequilibrium between loci $j$ and $k$ \\
\hline$V_{\mathrm{G}}$ & $\begin{array}{l}\text { genetic variance (variance of } z_{i} \text { among individuals, which at } \\
\text { equilibrium should be the same for all traits } i \text { ) }\end{array}$ \\
\hline$V_{\mathrm{g}}$ & genic variance (see Appendix A) \\
\hline
\end{tabular}

